\title{
205 - Building and Using an Apps Library for People Living with Dementia and their Carers
}

Maurice Mulvenna, School of Computing, Ulster University for Soo Hun, Digital Health \& Care Northern Ireland, Health and Social Care Board

Presentation 1: Apps Library as a digital resource for people living with dementia and carers; Soo Hun, Programme Manager, Digital Health \& Care Northern Ireland, Health and Social Care Board

Presentation 2: Development of a CLEAR Dementia Care (C) App; Frances Duffy, Consultant Lead Clinical Psychologist for Older People, Northern Health and Social Care Trust

Presentation 3: Technology Facilitated Reminiscence in Dementia. Assumpta Ryan, Professor of Ageing and Health, School of Nursing, Ulster University

Presentation 4: Understanding behaviour of people living with dementia using apps; Maurice Mulvenna, Professor of Computer Science, Raymond, Bond, Courtney Potts, School of Computing, Ulster University.

\section{Summary of Symposium}

The symposium describes the context and rationale behind the Health and Social Care Board Northern Ireland taking the strategic initiative to develop digital resources to support an 'app' library. The initiative, part of the Dementia eHealth and Data Analytics Pathfinder Programme is developing a digital framework to enable the social prescribing of apps, initially in support of those with dementia and their carers. In this symposium, apps that have been developed are described, including the CLEAR Dementia Care (C) App, developed to help carers understand behaviour from the perspective of the person with dementia, and the Inspire $\mathrm{D}$ app for individual specific reminiscence, for people living with dementia and their family carers. The final talk describes the new opportunities for researchers to employ anonymous digital data in their research studies to understand the behaviour of people living with dementia and their carers in using such digital apps.

\section{Presentation 1:}

Apps Library as a digital resource for people living with dementia and carers Soo Hun, Programme Manager, Digital Health \& Care Northern Ireland, Health and Social Care Board

People living with dementia and their carers need support to enable them to manage their condition and to live independently for as long as possible. Digital technology such as smartphones are owned by $79 \%$ of adults. There are over 300,000 health apps and $4 \mathrm{~m}$ apps are downloaded daily. A new digital service offering support for people living with dementia and their carers has been launched by the Health and Social Care Board Northern Ireland. The initiative, part of the Dementia eHealth and Data Analytics Pathfinder Programme, uses data and technology to inform better services, support people with dementia and help plan for the future. The aim of the apps4dementia library, https://apps4dementia.orcha.co.uk, is to provide a place for users to find safe, trusted mobile Apps to provide information and guidance on the condition, support self-care of symptoms and enable people affected by dementia and their carers; to carry on with their day-to-day activities for as long as possible. A series of engagement workshops conducted with people affected by dementia and carers provided input and feedback on the design, content and usability of the library; suitability and types of Apps for inclusion, as well as training materials to be delivered to healthcare professionals. Developed in conjunction with App evaluator, Organisation for the Review of Care and Health Apps (ORCHA), the library offers a range of applications which have been independently checked and reviewed for data privacy, clinical assurance and user experience. 10 best rated Apps are available on the library that provide guidance and information, to help with sleep, communication, keeping minds active, reminders 
as well as apps that support carers to care for their loved ones. The library will help to identify gaps in digital tools and enable commissioning of necessary Apps, of which 2 new apps - InspireD and CLEAR app will be included in the library in March 2020.

\section{Presentation 2:}

\section{Development of a CLEAR Dementia Care @ App}

Frances Duffy, Consultant Lead Clinical Psychologist for Older People, Northern Health and Social Care Trust

People living with dementia can present with behaviours that carers find difficult to understand. These behaviours are often a sign of distress the person experiences as they try to understand what is happening around them or when they are trying to meet their needs and are unable to tell others what they want. Failure to understand these behaviours can lead to increased distress for the person and their carers. It can also lead to unnecessary breakdown in placement or avoidable admission to hospital. CLEAR Dementia Care (C) was developed to help carers to understand behaviour from the perspective of the person with dementia. This helps carers to offer appropriate support which reduces distress and improves quality of life. The model has successfully implemented across a range of organisations.

A workshop attended by health care professionals, voluntary and community sector and people with dementia and their carers elicited positive feedback about the utility of a CLEAR Dementia Care App. Throughout development there has been Personal and Public Involvement (PPI) about the design and layout of the App from a range of potential service users. The outcome is an App that can easily be used by any person who has contact with a person with dementia, including family carers and paid staff. The app provides concise and accessible information to help understand behaviour and suggest ways which could reduce the distress associated with the behaviour. It provides useful information that is always easily available. This can enable early intervention to prevent escalation of behaviour, reduce distress to the person with dementia and also reducing carer stress and burnout. An additional function gives carers the option to chart behaviour in an easy and accessible way. This will provide further understanding about patterns in behaviour and help to identify potential causes.

Presentation 3:

\section{Technology Facilitated Reminiscence in Dementia}

Assumpta Ryan, Professor of Ageing and Health, School of Nursing, Ulster University

Reminiscence has been widely used as a therapeutic approach for people living with dementia and recent studies have focused on the use of technology to support the reminiscence experience. The aim of this study was to investigate the outcomes of a home based, individual specific reminiscence intervention facilitated through the use of an iPad app for people living with dementia and their family carers. The study used a quasi-experimental design, incorporating quantitative and qualitative components in three phases. Phase 1: A User Development Group comprising a paired sample of 6 people living with dementia and their family carers worked with the research team to refine and test the technology. Phase 2: The refined application was implemented with a paired sample of 30 people living with mild to moderate dementia and their family carers. All participants were provided with reminiscence and IT training and used the system for 12 weeks at home. Outcome measures, collected at three time points, examined the impact of reminiscence on mutuality, wellbeing, quality of life and quality of the relationship between participants living with dementia and their family carers. Phase 3 : Individual interviews were conducted with a sample of participants $(n=32)$ to explore their experience of the intervention. User interactions revealed that people living with dementia used the app independently and more frequently than their carers. There were statistically significant increases in mutuality, quality of caregiving relationships and emotional well-being from baseline to endpoint for people living with dementia. For carers, there were no significant changes in mutuality, quality of 
caregiving relationship and emotional wellbeing scores from baseline to endpoint. Participating dyads perceived the intervention as a positive experience which focused on gains rather than losses in the context of memory retention and learning new skills.

Presentation 4:

Understanding behaviour of people living with dementia using apps

Maurice Mulvenna, Professor of Computer Science, Raymond Bond, Courtney Potts, School of Computing, Ulster University.

Behavioural data analytics and event log analysis can be useful to gain insight into how users interact with technologies. Electronic event logging data were obtained for people living with dementia and family carers. Event logging is when each anonymised user interaction with an app is automatically logged and stored in a database. This study adopted the health interaction log data analysis pipeline, which involved data cleaning and preparation, as well as the use of exploratory data analysis and Kmeans clustering, to uncover behavioural patterns of usage by users of the InspireD reminiscence app, to address the question: What is the temporal behaviour of users interacting with the app? The results build on the findings of a feasibility study which found that that people living with dementia preferred to interact with photos and reminisce with personalized media. Reminiscing peaked on Thursdays and Fridays but dipped at the weekends. There was a correlation between the number of days the people living with dementia and carer interacted with the app. However, people living with dementia had many more interactions than carers. People living with dementia interacted with the app akin to one reminiscence session per week. K-means clustering uncovered four user archetypes, described as: a person living with dementia who demonstrates independent and consistent use of the app; a person living with dementia who is reliant on his/her carers for support with the app and exhibits unpredictable usage patterns; a person living with dementia who is highly reliant on his/her carers for engagement with the app; and very infrequent users. 